Metal-free plasma-enhanced chemical vapor deposition of large area nanocrystalline graphene

This content has been downloaded from IOPscience. Please scroll down to see the full text. 2014 Mater. Res. Express 1025031

(http://iopscience.iop.org/2053-1591/1/2/025031)

View the table of contents for this issue, or go to the journal homepage for more

Download details:

IP Address: 152.78.236.139

This content was downloaded on 30/07/2014 at 08:17

Please note that terms and conditions apply. 


\title{
Metal-free plasma-enhanced chemical vapor deposition of large area nanocrystalline graphene
}

\author{
Marek E Schmidt ${ }^{1}$, Cigang $\mathrm{Xu}^{2}$, Mike Cooke ${ }^{2}$, Hiroshi Mizuta ${ }^{1,3}$ and \\ Harold M H Chong ${ }^{3}$ \\ ${ }^{1}$ School of Materials Science, Japan Advanced Institute of Science and Technology (JAIST), \\ Nomi, Ishikawa 923-1292, Japan \\ ${ }^{2}$ Oxford Instruments Plasma Technology, North End, Yatton, Bristol, UK \\ ${ }^{3}$ Nano Research Group, School of Electronics and Computer Science, University of \\ Southampton, Highfield, Southampton, UK \\ E-mail: schmidtm@jaist.ac.jp
}

Received 6 March 2014, revised 16 April 2014

Accepted for publication 28 April 2014

Published 29 May 2014

Materials Research Express 1 (2014) 025031

doi:10.1088/2053-1591/1/2/025031

\begin{abstract}
This paper reports on large area, metal-free deposition of nanocrystalline graphene (NCG) directly onto wet thermally oxidized $150 \mathrm{~mm}$ silicon substrates using parallel-plate plasma-enhanced chemical vapor deposition. Thickness nonuniformities as low as $13 \%$ are achieved over the whole substrate. The cluster size $L_{\mathrm{a}}$ of the as-obtained films is determined from Raman spectra and lies between 1.74 and $2.67 \mathrm{~nm}$. The film uniformity was further confirmed by Raman mapping. The sheet resistance $R_{\mathrm{sq}}$ of $3.73 \mathrm{k} \Omega$ and charge carrier mobility $\mu$ of $2.49 \mathrm{~cm}^{2} \mathrm{~V}^{-1} \mathrm{~s}^{-1}$ are measured. We show that the NCG films can be readily patterned by reactive ion etching. NCG is also successfully deposited onto quartz and sapphire substrates and showed $>85 \%$ optical transparency in the visible light spectrum.
\end{abstract}

Keywords: PECVD, nanocrystalline graphene, wafer-scale

\section{Introduction}

Graphene, a one-atom thin carbon layer with hexagonal honeycomb structure, has recently attracted considerable research interest. It is considered a possible successor of silicon in the post-Moore era due to its unique mechanical and electronic properties [1]. However, the application of these extraordinary properties at commercial scale is hindered by the lack of appropriate methods to obtain large area, single crystal graphene. The exfoliation from highly oriented pyrolytic graphite yields the highest quality graphene so far, however, it does not scale and mechanical exfoliation at wafer-scale remains doubtful. Epitaxial growth on silicon carbide 
(SiC), on the other hand, allows good control over the number of layers, but the electrical properties of the films become dependent on the orientation due to the surface steps forming during growth, and the expensive $\mathrm{SiC}$ substrates have to be processed at temperatures exceeding $1200{ }^{\circ} \mathrm{C}$ [2]. The third method to be mentioned here is graphene growth on transition metal catalysts, such as $\mathrm{Ni}$ [3], $\mathrm{Cu}$ [4] and $\mathrm{Fe}$ [5] among others. The catalyst is exposed to a carbon-rich atmosphere at elevated temperature, resulting in the carbon to solve inside the catalyst and crystallizing on the surface upon cooling. The carbon source can be either obtained purely through thermal decomposition (chemical vapor deposition (CVD)) or with the help of a plasma (plasma-enhanced chemical vapor deposition (PECVD)) from hydrocarbon gases, such as methane $\left(\mathrm{CH}_{4}\right)$. To make the obtained graphene film usable for electronic applications it is necessary to transfer the film from the catalyst onto a different substrate. This method is being constantly developed and a roll-to-roll process has been recently demonstrated [6]. Nevertheless, there is still scope for research in direct deposition of graphene and nanocrystalline graphene (NCG) layer onto insulator substrates [7]. There has been some research into metal-free deposition of graphene to achieve this goal, such as molecular beam epitaxy (MBE) [8, 9], CVD [7, 10, 11] and various plasma-enhanced methods based on partially modified tools [12-14]. Although these films have typically a higher defect rate compared to graphene obtained by exfoliation or CVD, they can be suitable for applications such as saturable absorber in mode-locked lasers [15].

Here, we demonstrate large area synthesis of NCG on wet thermally oxidized silicon substrates, quartz glass and sapphire glass by a capacitively coupled parallel-plate PECVD process at high pressure of 1.5 Torr. We investigate the quality of these films deposited at different temperatures and conditions, and fabricate two terminal devices to study the resistivity.

\section{Deposition method and characterization}

NCG films were deposited on wet thermally oxidized $\left(100{ }^{\circ} \mathrm{C}, 48 \mathrm{~min}, 244 \mathrm{~nm}\right.$ oxide thickness) n-Si wafers with a diameter of $150 \mathrm{~mm}$. We use an Oxford Instruments Nanofab 1000 Agile in this work, which can process substrates with up to $200 \mathrm{~mm}$ diameter. The deposition chamber is schematically illustrated in figure 1(a). The sample is placed on the electrically grounded, temperature controlled lower electrode (graphite, up to $1000{ }^{\circ} \mathrm{C}$ ) which is used together with the upper electrode to generate the capacitively coupled plasma. The process gas is introduced uniformly through holes in the upper electrode.

The deposition process is illustrated in figure 1(b). First, the substrate is loaded into the process chamber at the loading temperature $T_{\text {load }}=690^{\circ} \mathrm{C}$ and heated to the deposition temperature $T_{\text {dep }}$. Next, the substrate is cleaned at $T_{\text {dep }}$ by introduction of $\mathrm{H}_{2}$ gas $(200 \mathrm{sccm}, 1000$ mTorr) for typically $t_{\text {clean }}=15 \mathrm{~min}$. In the deposition step, $\mathrm{CH}_{4}$ and $\mathrm{H}_{2}$ are introduced (1500 $\mathrm{mT}$ pressure) and the RF plasma is generated (100 W RF) for $t_{\text {dep }}$. Finally, the substrate is cooled down to $T_{\text {load }}$ and unloaded. In case $T_{\text {dep }}$ is below $690^{\circ} \mathrm{C}$ the heating and cooling steps are omitted.

A total of eight wafers were deposited with varying process parameters, with the relevant conditions summarized in table 1 . Between wafer 1 and wafer 3 only the methane flow rate $F\left[\mathrm{CH}_{4}\right]$ was varied while keeping all other parameters fixed. Then, the effect of the deposition temperature was varied for wafers 4-7. Finally, wafer 8 was deposited to finalize the deposition 
a) shower head process
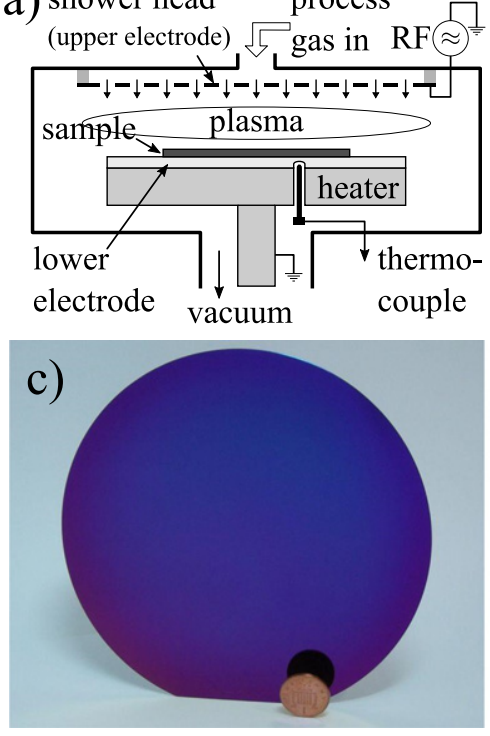

b) Temperature

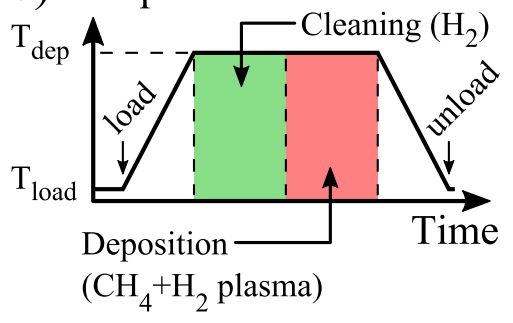

d)

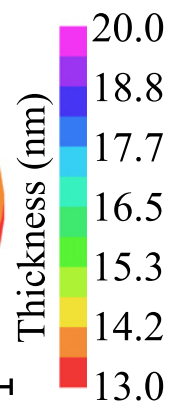

Figure 1. (a) Schematic representation of deposition chamber. (b) Illustration of deposition process. (c) Photograph of thermally oxidized $150 \mathrm{~mm}$ silicon wafer after PECVD deposition of NCG. (d) Thickness distribution of NCG film on different $150 \mathrm{~mm}$ wafer obtained by ellipsometry.

Table 1. Deposition conditions and corresponding measurement results.

\begin{tabular}{lcccccccc}
\hline & $\begin{array}{c}T_{\text {dep }} \\
\left({ }^{\circ} \mathrm{C}\right)\end{array}$ & $\begin{array}{c}t_{\text {dep }} \\
(\mathrm{min})\end{array}$ & $\begin{array}{c}F\left[\mathrm{H}_{2}\right] \\
(\mathrm{sccm})\end{array}$ & $\begin{array}{c}F\left[\mathrm{CH}_{4}\right] \\
(\mathrm{sccm})\end{array}$ & $\begin{array}{c}N U^{100} \\
(\%)\end{array}$ & $\begin{array}{c}d_{\text {mean }}^{100} \\
(\mathrm{~nm})\end{array}$ & $\begin{array}{c}\mathrm{rms} \\
(\mathrm{nm})\end{array}$ & $\begin{array}{c}L_{\mathrm{a}} \\
(\mathrm{nm})\end{array}$ \\
\hline Wafer 1 & 900 & 15 & 75 & 60 & 7 & 35.5 & 2.04 & $1.79 \pm 0.01$ \\
Wafer 2 & 900 & 15 & 75 & 30 & 6 & 31.5 & 2.25 & $1.81 \pm 0.01$ \\
Wafer 3 & 900 & 15 & 75 & 10 & 7 & 18.0 & 1.17 & $2.06 \pm 0.03$ \\
Wafer 4 & 900 & 5 & 75 & 60 & 4 & 15.7 & 1.27 & $1.74 \pm 0.02$ \\
Wafer 5 & 850 & 5 & 75 & 60 & 6 & 12.4 & 0.91 & $1.83 \pm 0.02$ \\
Wafer 6 & 800 & 5 & 75 & 60 & 11 & 8.9 & 0.56 & $2.09 \pm 0.02$ \\
Wafer 7 & 750 & 5 & 75 & 60 & 25 & 5.2 & 0.41 & $2.39 \pm 0.02$ \\
Wafer 8 & 900 & 1 & 75 & 60 & 7 & 3.0 & 0.23 & $2.67 \pm 0.06$ \\
\hline
\end{tabular}

duration series (together with wafer 1 and 4). A photograph of a $150 \mathrm{~mm}$ wafer with deposited NCG is shown in figure $1(\mathrm{c})$.

The film thickness of the as-deposited substrates is then mapped using a Woollam M-2000 ellipsometer ( $5 \mathrm{~mm}$ offset from edge, 180 data points), with the result for Wafer 4 shown in figure 1(d). The accuracy of the ellipsometer measurement was confirmed to be $\pm 10 \%$ through atomic force microscopy measurement of surface steps created by oxygen RIE on reference samples.

Raman spectra of carbon films are well understood and contain a large amount of information [16, 17]. From the ellipsometer measurement shown in figure 1(d) it is apparent that there is a slight non-uniformity across the $150 \mathrm{~mm}$ substrates, which is expected to be also prominent in the Raman spectra. We therefore performed Raman mapping using a Renishaw inVia Raman microscope. However, due to the limited travel range of the motorized sample 
stage of the Raman spectrometer, $40 \times 40 \mathrm{~mm}^{2}$ samples were first extracted through dicing (deposited film facing dicing tape) and used for the Raman mapping. The location of these quadratic samples is indicated in figure 1(d) by dashed lines. A total of 64 Raman spectra $(5 \mathrm{~mm}$ spacing) were acquired on each sample using $532 \mathrm{~nm}$ excitation wavelength.

The surface topography was measured in an $1 \times 1 \mu \mathrm{m}$ large area close to the center of each of the wafers by contact mode AFM in order to obtain the root mean square (rms) roughness values.

In order to measure the electrical properties of the films, simple two-terminal devices were fabricated. First, optical lithography was used to pattern $60 \mu \mathrm{m}$ wide lines with lengths of 80 , 280,500 and $680 \mu \mathrm{m}$ by photoresist, followed by an $\mathrm{O}_{2}$ reactive ion etching step (20 W RF power, $20 \mathrm{mT}$ pressure, $20 \mathrm{sccm} \mathrm{O}_{2}$ gas flow).

\section{Results and discussion}

\subsection{Ellipsometer}

One of the key aspects of the reported deposition process is the uniformity across the whole substrate. Therefore, the non-uniformity $N U$ of the thickness distribution obtained through ellipsometry was calculated by $N U=(\max (d)-\min (d)) /(\max (d)+\min (d)) \times 100 \%$. First, all 180 data points were considered, resulting in non-uniformity values between $13 \%$ (wafer 4) and 92\% (wafer 1). The latter value was caused by several extreme data points at the edge of the wafer caused by local contamination. Additionally, the non-uniformity was calculated for the data points within the inner $100 \mathrm{~mm}$ diameter circle to compensate for the edge non-uniformity observed in the ellipsometer results such as the one shown in figure 1(d) (wafer 3). These non-uniformity values for the $100 \mathrm{~mm}$ equivalent $N U^{100}$ are significantly lower for all wafers with the largest drop for wafer 1 from $92 \%$ to $7 \%$ (the $N U^{100}$ values are listed in table 1). A certain non-uniformity is common for plasma processes, as the plasma, temperature or gas distribution can suffer from edge effects. The cause in this case was not further investigated. Additionally, the mean thickness was calculated from the ellipsometer results in the $100 \mathrm{~mm}$ equivalent area and are shown as $d_{\text {mean }}^{100}$ in table 1 . There is a significant increase of non-uniformity with decreasing temperature which was not further investigated.

\subsection{Raman}

A representative Raman spectrum obtained on wafer 2 is shown in figure 2 (blue curve). Curve fitting was used to determine the position and intensity of the individual peaks (red curve). Here, we used Lorentzian curve shapes for all peaks with the exception of the D' peak (1627 $\mathrm{cm}^{-1}$ ), which was fitted by a Fano line shape [18]. The individual components of the fit model are shown as black curves. The spectrum exhibits distinct D, G and 2D peaks, which are characteristic for defective graphene [17]. Other peaks observed in this spectrum, such as the D', D+D', D+D' and 2D' (not shown) had been reported for carbon films before, and are briefly explained in table 2 alongside the other three peaks.

Since the spectrum exhibits a $D$ peak with an intensity larger than the $G$ and 2D peaks, the films obtained in this work have a significant number of crystal defects in comparison to graphene or graphite. The intensity ratio of the D-peak to G-peak $I(D) / I(G)$ is $\approx 2.7$ for the 


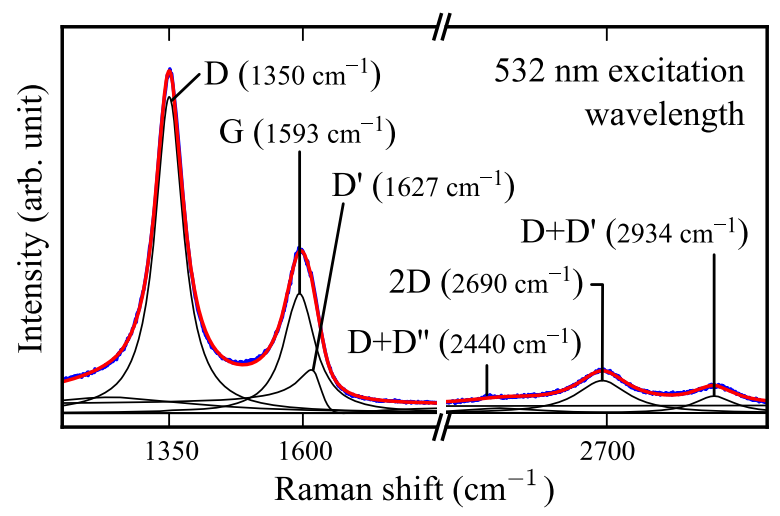

Figure 2. Raman spectrum obtained on $30 \mathrm{~nm}$ thick NCG film deposited at $900{ }^{\circ} \mathrm{C}$. The peaks are explained in table 2 .

Table 2. List of Raman peaks observed in NCG spectra (such as the one shown in figure 2), together with Raman shift and function used for fitting; LO for Lorentzian and FAN for Fano line-shape.

\begin{tabular}{|c|c|c|}
\hline Peak & Shift & Description \\
\hline $\mathrm{D}$ & 1350 & $\begin{array}{l}\text { [LO] Intervalley defect }\left(\mathrm{A}_{1 \mathrm{~g}} \text {-mode), present only in disordered }\right. \\
\text { graphene or edge areas. }\end{array}$ \\
\hline G & 1593 & [LO] Radial breathing mode of sp2 carbon forms $\left(\mathrm{E}_{2 \mathrm{~g}}-\right.$ mode $)$. \\
\hline $\mathrm{D}^{\prime}$ & 1627 & [FAN] Intravalley defect $[16,19]$ \\
\hline $\mathrm{D}+\mathrm{D}^{\prime \prime}$ & 2441 & $\begin{array}{l}\text { [LO] Intervalley double resonance process; sometimes referred to } \\
\text { as } \mathrm{G}^{*}[16,20]\end{array}$ \\
\hline $2 \mathrm{D}$ & 2690 & $\begin{array}{l}\text { [LO] Sum of double and triple resonances, sometimes referred to as } \\
\mathrm{G}^{\prime}[16,19,21]\end{array}$ \\
\hline $\mathrm{D}+\mathrm{D}^{\prime}$ & 2934 & [LO] Defect related band, sometimes referred to as $\mathrm{D}+\mathrm{G}$. $[19,21]$ \\
\hline $2 \mathrm{D}^{\prime}$ & 3248 & $\begin{array}{l}\text { [LO] (Not visible in figure 2) Overtone of } \mathrm{D}^{\prime} \text {, sometimes referred } \\
\text { to as } \mathrm{G}^{\prime \prime} .[21,22]\end{array}$ \\
\hline
\end{tabular}

shown spectrum. Furthermore, the G-peak location is not significantly affected by the excitation wavelength (confirmed for 532, 633 and $785 \mathrm{~nm}$ ). These characteristics $(I(\mathrm{D}) / I(\mathrm{G}) \approx 2, \operatorname{Pos}(\mathrm{G})$ $\approx 1590 \mathrm{~cm}^{-1}$ and $\operatorname{Pos}(\mathrm{G})$ unaffected by excitation wavelength) had been described previously and attributed to NCG [23]. Several recent publications reported on carbon films with very similar Raman spectra $[13,14]$ labeling them NCG or nanographene. Therefore, based on the Raman spectrum of our films and the current naming convention of thin carbon films in the scientific community, we label our films NCG.

The cluster diameter of the NCG can be calculated from the D and G peak intensities as $L_{\mathrm{a}}=(I(\mathrm{G}) \cdot C(\lambda)) / I(\mathrm{D})$ with $C(\lambda)$ a variable scaling coefficient which depends on the excitation wavelength [24]. $L_{\mathrm{a}}$ for the spectrum shown in figure 2 is thus $1.81 \mathrm{~nm}$, and the values of the remaining wafers (spectra obtained close to center of wafers) are listed in table 1 .

As mentioned earlier, we also performed Raman mapping. The obtained spectra were analyzed as explained above, resulting in the maps shown in figures 3(a), (b) and (c) (wafer 3, 

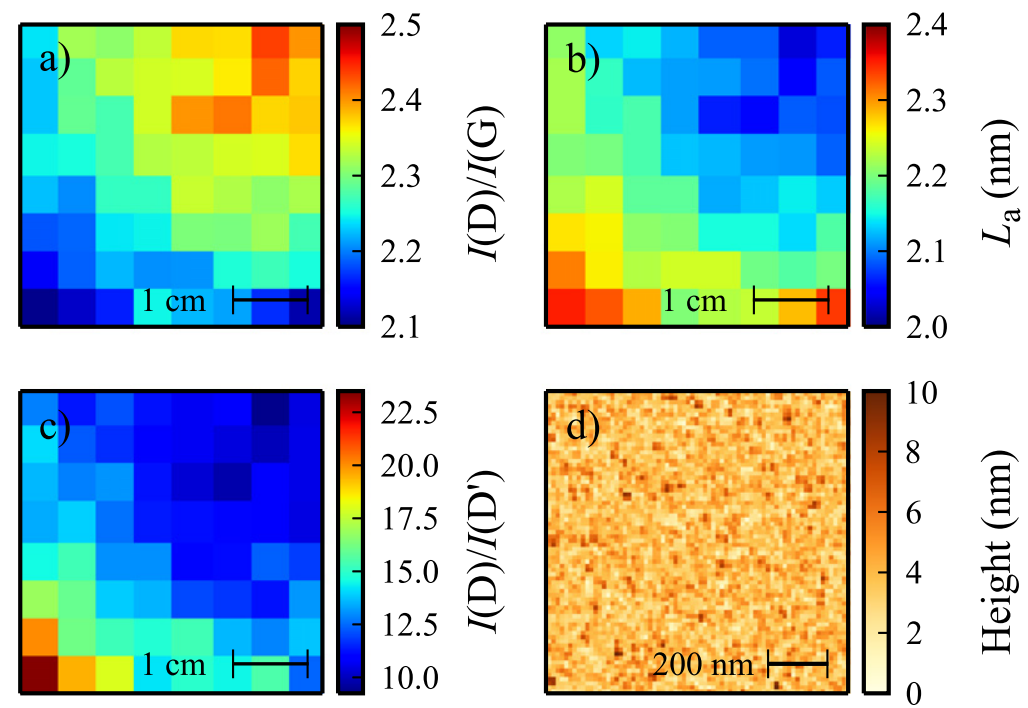

Figure 3. (a)+(c) Raman mapping results of area indicated in figure 1(d). (b) Cluster size derived from $I(\mathrm{D}) / I(\mathrm{G})$ map shown in (a). (d) Topography of typical NCG film acquired by AFM.

top right data point at center of wafer). The concentric pattern seen in the thickness distribution (compare figure 1(d)) is also clearly visible in all Raman results.

The $I(\mathrm{D}) / I(\mathrm{G})$ ratio map (figure 3(a)) and the derived $L_{\mathrm{a}}$ map (figure $3(\mathrm{~b})$ ) show that the cluster diameter is smallest at the center of the wafer, the place of the largest thickness. This trend was observed for all wafers deposited in this work. Furthermore, we did not find any evidence of a correlation between deposition temperature and cluster diameter. The $I(\mathrm{D}) / I\left(\mathrm{D}^{\prime}\right)$ ratio map (figure 3(c)) shows a decrease towards the center of the wafer. A ratio of 10 indicates a mix of vacancy-like and sp3 defects, while a ratio of 13 and above shows a prevalence of sp3 defects [18].

\subsection{Surface roughness}

The Raman spectra clearly indicate nanocrystalline structure, however, it is also important to know the roughness of the films in relation to the cluster size. Therefore, atomic force microscopy in contact mode was used to measure the topography of $1 \times 1 \mu \mathrm{m}$ areas close to the center of the wafers. The result for wafer 3 is shown in figure 3(d), indicating that the surface comprises round particles. The average distance between the particles was manually extracted from the AFM data and is between 26 and $37 \mathrm{~nm}$ ( $\sim 27 \mathrm{~nm}$ for wafer 3), much larger compared to the respective cluster diameters. This observation suggests that the observable surface topography is not related to the cluster diameter. The extracted rms roughness values for all of the wafers are listed in table 1 . These rms values are between 5 and $8 \%$ of the film thickness, independent of process conditions or film thickness.

\subsection{Optical}

NCG films were also deposited directly onto small quartz glass and sapphire glass samples $\left(T_{\text {dep }}=900^{\circ} \mathrm{C}, t_{\text {dep }}=15 \mathrm{~min}\right)$. A molybdenum disk was used as handle wafer for the deposition 


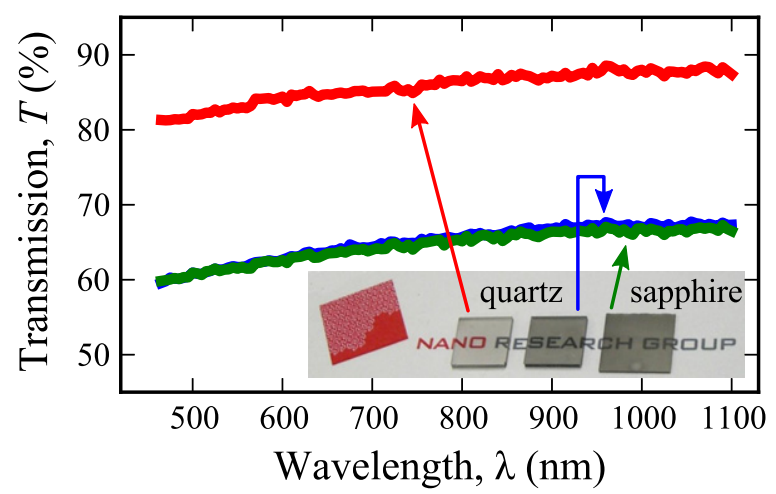

Figure 4. Measured optical transmission of the quartz and sapphire samples shown in the inset. Left: quartz with $5.5 \mathrm{~nm}$ NCG, middle: quartz with $15 \mathrm{~nm}$ NCG, right: sapphire with $15 \mathrm{~nm}$ NCG.

due to its higher thermal conductivity compared to $\mathrm{Si}$. To obtain the film transmittance, substrate absorption and film reflection were measured separately and accounted for. Three samples are shown in figure 4 together with their film transmittance across the visible range. The left quartz glass sample with $5.5 \mathrm{~nm}$ thick film $\left(F\left[\mathrm{H}_{2}\right]=60 \mathrm{sccm}, F\left[\mathrm{CH}_{4}\right]=75 \mathrm{sccm}\right)$ offers $85 \%$ transparency and $13 \mathrm{k} \Omega$ sheet resistance at $550 \mathrm{~nm}$ wavelength, while the middle quartz glass sample $\left(F\left[\mathrm{H}_{2}\right]=72 \mathrm{sccm}, F\left[\mathrm{CH}_{4}\right]=90 \mathrm{sccm}\right)$ with $15 \mathrm{~nm}$ film thickness has only $65 \%$ transparency but much lower $2.5 \mathrm{k} \Omega$ sheet resistance (approximately linear decrease of transparency with NCG thickness). The sapphire sample, deposited with identical conditions as the middle quartz glass sample, has also a $15 \mathrm{~nm}$ thick NCG film and $65 \%$ transparency, however, a much higher sheet resistance of $7.5 \mathrm{M} \Omega$. The reason for this high resistivity was not further investigated. The sheet resistance values are considerably higher than the values reported for mono- and bi-layer graphene $[2,25]$, however the reported deposition technique has not been optimized for optical electrode applications and the deposition does not require a transfer step as is the case for CVD grown graphene. The slight decrease of transparency at lower wavelengths is also observed in graphene obtained by other methods $[25,26]$.

\subsection{Electrical characteristics}

In order to measure the electrical characteristics of the newly developed NCG films, test structures were fabricated on a reference sample $\left(T_{\text {dep }}=900^{\circ} \mathrm{C}, t_{\text {dep }}=15 \mathrm{~min}, F\left[\mathrm{H}_{2}\right]=90 \mathrm{sccm}\right.$, $F\left[\mathrm{CH}_{4}\right]=72 \mathrm{sccm}$ ) by using standard thin film fabrication techniques. $60 \mu \mathrm{m}$ wide lines were first patterned by optical lithography and oxygen based reactive ion etching (Oxford Instruments RIE80, $20 \mathrm{sccm} \mathrm{O}_{2}, 20 \mathrm{mTorr}$ chamber pressure, $20 \mathrm{~W}$ RF power), followed by probe pad patterning using a titanium/gold lift-off process. An SEM micrograph of such a fabricated device is shown as inset of figure 5. Consecutively, the $I_{\mathrm{D}} / V_{\mathrm{D}}$ characteristics were measured using an Agilent B1500A semiconductor device parameter analyzer. All devices showed metallic-like $I_{\mathrm{D}} / V_{\mathrm{D}}$ characteristics between 273 and $79 \mathrm{~K}$ as shown in the upper left inset of figure 5 for a $80 \mu \mathrm{m}$ long device. The increasing resistivity with decreasing temperature is common for semiconducting materials. The device resistances measured at room temperature are plotted against the channel lengths in figure 5 . The contact resistance of $0.69 \mathrm{k} \Omega$ is obtained by linear fitting, and the sheet resistance $R_{\mathrm{sq}}=9.3 \pm 1.9 \mathrm{k} \Omega \mathrm{sq}^{-1}$ is calculated. The charge 


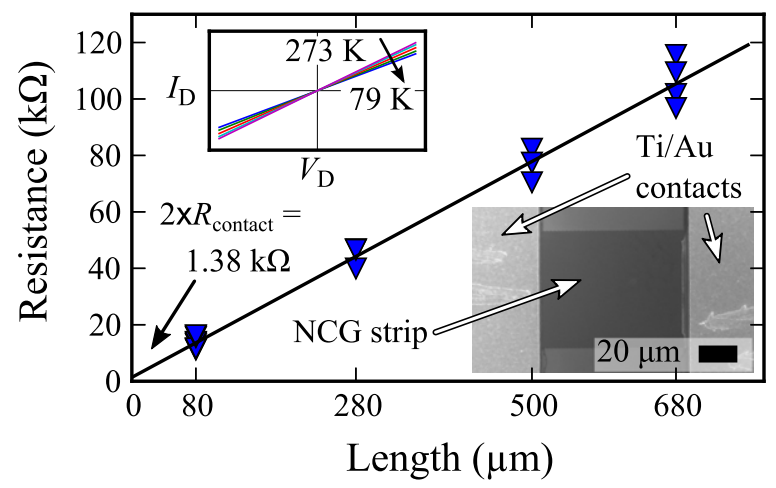

Figure 5. Measured resistance for NCG strips with various lengths. SEM micrograph of NCG strips contacted by Ti/Au lift-off shown as inset lower-right, and the $I_{\mathrm{D}} / V_{\mathrm{D}}$ characteristics of an $80 \mu \mathrm{m}$ long device for temperatures between 273 and $79 \mathrm{k}$ are shown as inset upper left.

carrier mobility $\mu$ of $2.49 \mathrm{~cm}^{2} \mathrm{~V}^{-1} \mathrm{~s}^{-1}$ was measured using an Accent HL5500 Hall System, alongside with the sheet electron concentration of $n_{\mathrm{s}}=6.73 \times 10^{14} \mathrm{~cm}^{-2}$. This corresponds to a bulk electron concentration of $n=2.22 \times 10^{20} \mathrm{~cm}^{-3}$.

The sheet resistance and charge carrier mobility are in a very similar range as values reported for NCG elsewhere. The films deposited by Kalita [13] using surface wave plasmaenhanced chemical vapor deposition (PECVD) have sheet resistances of 3-7 k $\Omega \mathrm{sq}^{-1}$. Charge carrier mobility and concentration were not reported. The ECR-CVD deposited films at $400^{\circ}$ by Medina [27] have sheet resistances in the range of $5-20 \mathrm{k} \Omega \mathrm{sq}^{-1}$ without reporting the mobility or concentration of charge carrier. The charge carrier mobility of $\mu=1 \mathrm{~cm}^{2} \mathrm{~V}^{-1} \mathrm{~s}^{-1}$, as reported for MBE grown films on sapphire, is slightly lower than the values reported here, however no sheet resistance is provided [8].

\section{Conclusion}

Metal-free deposition of large area NCG using a parallel plate plasma-enhanced CVD tool was demonstrated. The deposition was carried out at temperatures between 750 and $900^{\circ}$. The high uniformity of the film thickness across the whole $150 \mathrm{~mm}$ substrates was demonstrated, and the domain size of these graphene based films were studied in detail by Raman spectroscopy. The cluster diameter $L_{\mathrm{a}}$ increases for thinner films, suggesting that the initially deposited films have a higher crystallinity. No correlation between deposition temperature and cluster diameter was observed.

The deposition of NCG on insulating, transparent substrates such as quartz glass or sapphire glass was demonstrated. The metallic electrical properties and the demonstrated patterning by dry oxygen etching make these films suitable for transparent electrode applications or wafer-scale electronic applications. 


\section{Acknowledgments}

The corresponding author would like to acknowledge the supported by the University of Southampton, School of Electronics and Computer Science Scholarship, and the Southampton Nanofabrication Centre.

\section{References}

[1] Novoselov K S, Geim A K, Morozov S V, Jiang D, Zhang Y, Dubonos S V, Grigorieva I V and Firsov A A 2004 Electric field effect in atomically thin carbon films Science 306 666-9

[2] Hass J, de Heer W A and Conrad E H 2008 The growth and morphology of epitaxial multilayer graphene J. Phys.: Condens. Matter 20323202

[3] Reina A, Jia X, Ho J, Nezich D, Son H, Bulovic V, Dresselhaus M S and Kong J 2009 Large area, Few-Layer graphene films on arbitrary substrates by chemical vapor deposition Nano Lett. 9 30-35

[4] Li X, Magnuson C W, Venugopal A, Tromp R M, Hannon J B, Vogel E M, Colombo L and Ruoff R S 2011 Large-area graphene single crystals grown by low-pressure chemical vapor deposition of methane on copper J. Am. Chem. Soc. 13328169

[5] Kondo D, Sato S, Yagi K, Harada N, Sato M, Nihei M and Yokoyama N 2010 Low-Temperature synthesis of graphene and fabrication of Top-Gated field effect transistors without using transfer processes Appl. Phys. Express 3025102

[6] Bae S et al 2010 Roll-to-roll production of 30-inch graphene films for transparent electrodes Nat. Nanotechnology 5 574-8

[7] Sun J, Lindvall N, Cole M T, Teo K B K and Yurgens A 2012 Chemical vapor deposition of nanocrystalline graphene directly on arbitrary high-temperature insulating substrates in 2012 7th IEEE Int. Conf. on Nano/ Micro Engineered and Molecular Systems (NEMS) (March) pp 11-14

[8] Jerng S K, Yu D S, Kim Y S, Ryou J, Hong S, Kim C, Yoon S, Efetov D K, Kim P and Chun S H 2011 Nanocrystalline graphite growth on sapphire by carbon molecular beam epitaxy J. Phys. Chem. C 115 $4491-4$

[9] Oliveira M H Jr, Schumann T, Gargallo-Caballero R, Fromm F, Seyller T, Ramsteiner M, Trampert A, Geelhaar L, Lopes J M J and Riechert H 2013 Mono-and few-layer nanocrystalline graphene grown on Al2O3(0001) by molecular beam epitaxy Carbon 56 339-50

[10] Rümmeli M H, Bachmatiuk A, Scott A, Börnert F, Warner J H, Hoffman V, Lin J-H, Cuniberti G and Büchner B 2010 Direct low-temperature nanographene CVD synthesis over a dielectric insulator ACS Nano 4 4206-10

[11] Sun J, Cole M T, Lindvall N, Teo K B K and Yurgens A 2012 Noncatalytic chemical vapor deposition of graphene on high-temperature substrates for transparent electrodes Appl. Phys. Lett. 100022102

[12] Baraton L, Gangloff L, Xavier S, Cojocaru C S, Huc V, Legagneux P, Lee Y H and Pribat D 2009 Growth of graphene films by plasma enhanced chemical vapour deposition Proc. SPIE 7399, Carbon Nanotubes, Graphene and Associated Devices II (San Diego, 18 August 2009) 73990 T

[13] Kalita G, Kayastha M S, Uchida H, Wakita K and Umeno M 2012 Direct growth of nanographene films by surface wave plasma chemical vapor deposition and their application in photovoltaic devices RSC Adv. 2 $3225-30$

[14] Zhang L, Shi Z, Wang Y, Yang R, Shi D and Zhang G 2011 Catalyst-free growth of nanographene films on various substrates Nano Res. 4 315-21

[15] Zheng Z, Zhao C, Lu S, Chen Y, Li Y, Zhang H and Wen S 2012 Microwave and optical saturable absorption in graphene Opt. Express 20 23201-14 
[16] Malard L M, Pimenta M A, Dresselhaus G and Dresselhaus M S 2009 Raman spectroscopy in graphene Phy. Rep. 473 51-87

[17] Ferrari A C and Basko D M 2013 Raman spectroscopy as a versatile tool for studying the properties of graphene Nat. Nanotechnology 8 235-46

[18] Eckmann A, Felten A, Mishchenko A, Britnell L, Krupke R, Novoselov K S and Casiraghi C 2012 Probing the nature of defects in graphene by raman spectroscopy Nano Lett. 12 3925-30

[19] Martins Ferreira E H, Moutinho M V O, Stavale F, Lucchese M M, Capaz R B, Achete C A and Jorio A 2010 Evolution of the raman spectra from single-, few-, and many-layer graphene with increasing disorder Phys. Rev. B 82125429

[20] Venezuela P, Lazzeri M and Mauri F 2011 Theory of double-resonant raman spectra in graphene: intensity and line shape of defect-induced and two-phonon bands Phys. Rev. B 84035433

[21] Dresselhaus M S, Jorio A, Souza Filho A G and Saito R 2010 Defect characterization in graphene and carbon nanotubes using raman spectroscopy Philos. Trans. R. Soc. A 368 5355-77

[22] Nemanich R J and Solin S A 1979 First-and second-order raman scattering from finite-size crystals of graphite Phys. Rev. B 20 392-401

[23] Ferrari A C and Robertson J 2004 Raman spectroscopy of amorphous, nanostructured, diamond-like carbon, and nanodiamond Philos. Trans. R. Soc. A 362 2477-512

[24] Ferrari A C and Robertson J 2000 Interpretation of Raman spectra of disordered and amorphous carbon Phys. Rev. B 6114095

[25] Bonaccorso F, Sun Z, Hasan T and Ferrari A C 2010 Graphene photonics and optoelectronics Nat. Photonics 4 611-22

[26] Kim K S, Zhao Y, Jang H, Lee S Y, Kim J M, Kim K S, Ahn J-H, Kim P, Choi J-Y and Hong B H 2009 Large-scale pattern growth of graphene films for stretchable transparent electrodes Nature 457 706-10

[27] Medina H, Lin Y-C, Jin C, Lu C-C, Yeh C-H, Huang K-P, Suenaga K, Robertson J and Chiu P-W 2012 Metal-free growth of nanographene on silicon oxides for transparent conducting applications Adv. Funct. Mater. 22 2123-8 